\title{
Testing QCD in the non-perturbative regime
}

A.W. Thomas

Jefferson Laboratory, 12000 Jefferson Avenue, Newport News, VA 23606, USA

e-mail: awthomas@jlab.org

\section{Introduction}

This is an exciting time for strong interaction physics. We have a candidate for a fundamental theory, namely QCD, which has passed all the tests thrown at it in the perturbative regime. In the non-perturbative regime it has also produced some promising results and recently a few triumphs but the next decade will see enormous progress in our ability to unambiguously calculate the consequences of non-perturbative QCD and to test those predictions experimentally. Amongst the new experimental facilities being constructed, the hadronic machines at JPARC and GSI-FAIR and the 12 GeV Upgrade at Jefferson Lab, the major new electromagnetic facility worldwide, present a beautifully complementary network aimed at producing precise new measurements which will advance our knowledge of nuclear systems and push our ability to calculate the consequences of QCD to the limit.

We will first outline the plans at Jefferson Lab for doubling the energy of CEBAF. The new facility presents some wonderful opportunities for discovery in strong interaction physics, as well as beyond the standard model. Then we turn to the theoretical developments aimed at extracting precise results for physical hadron properties from lattice QCD simulations. This discussion will begin with classical examples, such as the mass of the nucleon and $\rho$, before dealing with a very recent and spectacular success involving information extracted from modern parity violating electron scattering.

\section{The $12 \mathrm{GeV}$ upgrade at Jefferson Lab}

The Continuous Electron Beam Accelerator Facility (CEBAF) was designed to permit exploration of the detailed structure of nucleons and nuclei using continuous beams of 4 $\mathrm{GeV}$ electrons. Nuclear physics research at CEBAF started in 1995. The accelerator represents the world's first successful large-scale utilization of superconducting radiofrequency (SRF) accelerating structures. Advances in SRF technology have made it possible to increase the beam energy to $6 \mathrm{GeV}$ without installing new equipment. Further developments have led to performance that will permit us to increase the energy to $12 \mathrm{GeV}$ (double the present energy and triple the original) by installing a small number of new accelerating structures but without changing the basic configuration of the facility. Design work is presently underway for all systems. Research at $12 \mathrm{GeV}$ is to begin around 2012 . We begin by describing the accelerator upgrade plans as well as the experimental equipment planned to exploit the new research opportunities.

\subsection{Accelerator upgrade}

A new $12 \mathrm{GeV}$ beam to Hall D and beams of up to $11 \mathrm{GeV}$ for Halls $A, B$, and C are needed for the desired research program. To provide them, the accelerator must be upgraded to $2.2 \mathrm{GeV} /$ pass $(1.1 \mathrm{GV} /$ linac) and the beam transport system upgraded and expanded. The following summarizes the planned work:

- $1.1 \mathrm{GV} /$ linac: Increase each linac's voltage by $0.5 \mathrm{GV}$ by adding 5 new highperformance superconducting radio-frequency (SRF) accelerating systems and 
new RF system for each; the present $5 \mathrm{~kW} @ 2 \mathrm{~K}$ cryogenics plant will be roughly doubled to support the increased load;

- Add a tenth recirculation arc, thereby permitting a sixth pass of acceleration through the North Linac and direction of beam toward Hall D;

- Upgrade the capabilities of the present beam transport system to handle the increased beam energies; there will be extensive re-use of existing hardware;

- Add beam transport to Hall D.

\section{$6 \mathrm{GeV} \Rightarrow 12 \mathrm{GeV}$}

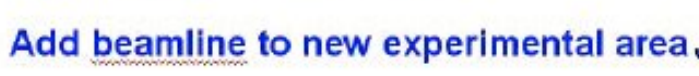

Add beamline to new experimental area Add 5 cryomodules 20 cryomodules

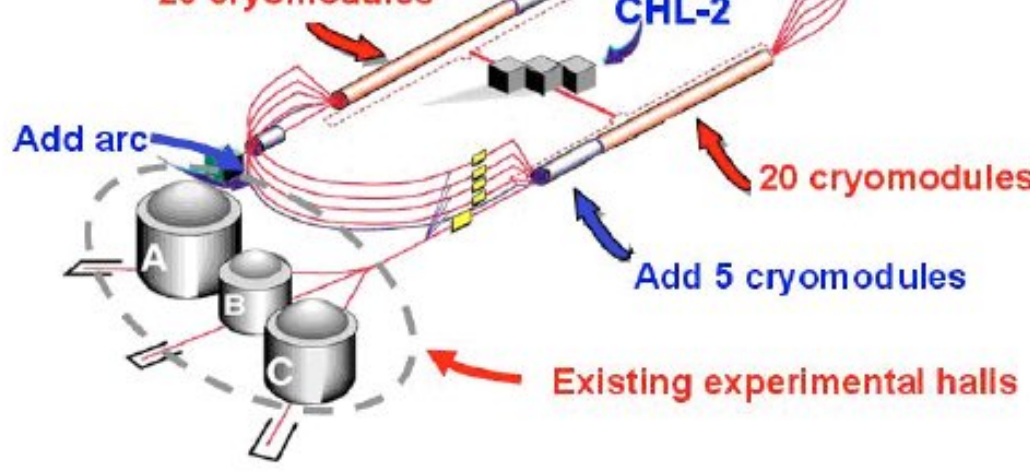

Add new experimental

area

Upgrade magnets and power supplies

Fig. 1. Illustration of the changes to the accelerator facility associated with the Upgrade of CEBAF from 6 to $12 \mathrm{GeV}$.

\subsection{Gluex experiment in Hall D}

Hall D will initially house the equipment for the GlueX experiment and will be situated at the northeast corner of the CEBAF accelerator. The design, fabrication and installation of detector components will be performed. System integration and checkout will be performed, including interfacing of detector systems to trigger, data acquisition, slow controls, and monitoring systems. Features of this nearly-hermetic spectrometer include:

- A large solenoid magnet, which in concert with large-area drift chambers provides momentum and vertex measurements for multiple charged particles;

- Reconstruction capability for energy and direction of multiple photons using large-area calorimetry;

- Particle identification for charged particles via time-of-flight, electromagnetic shower calorimeters, Cerenkov detectors, and energy loss;

- A facility for producing a beam of polarized photons of known energies;

- A data acquisition system capable of handling data from a flux of 107 photons per second on a hydrogen target, with an architecture which is capable of being upgraded to ten times higher capacity. 


\subsection{Hall $A$, $B$ and $C$ equipment}

- In Hall A the beamline will be upgraded to achieve the capability of delivering the maximum energy 5-pass beam to the existing spectrometers. Some electronics will also be upgraded to improve data-taking at higher data acquisition rates.

- In Hall B CLAS, the existing large acceptance detector in Hall B will be extensively upgraded with new magnets and detectors to capture the more forward-focused reaction products at the increased luminosity. Features of this upgraded spectrometer include:

o Toroidal magnetic field, which in concert with large-area drift chambers and silicon vertex detectors provides momentum and vertex measurements for multiple charged particles;

o Reconstruction of energy and direction of multiple photons in the forward direction using large-area calorimetry;

o Identification of charged particles via time-of-flight, electromagnetic shower calorimeters, and Cerenkov detectors;

- Flexibility to accommodate a variety of target types; and

- Magnetic shielding of Moller electrons adequate to permit measurements with a luminosity of $1035 \mathrm{~cm}-2 \mathrm{~s}-1$, and

- A data acquisition system capable of absorbing the resulting data.

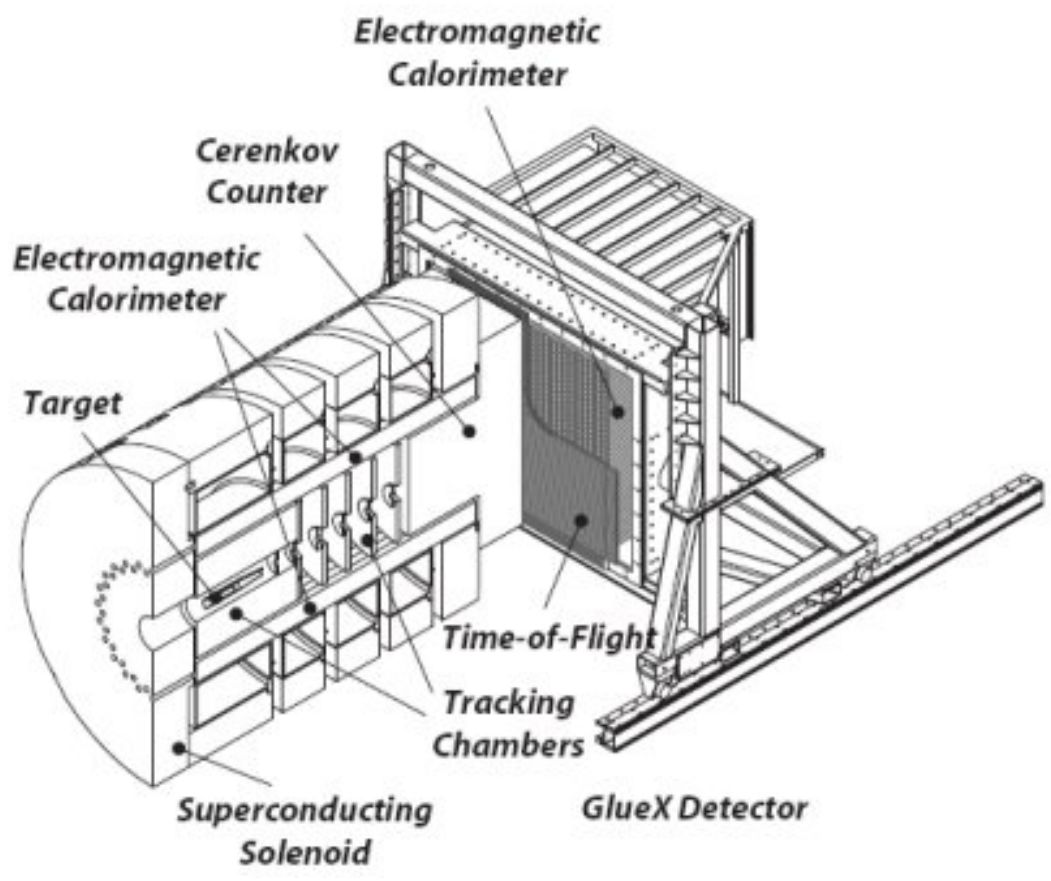

Fig. 2. The Gluex detector, specifically optimized to permit reliable partial wave analysis for multimeson final states, to be installed in Hall D. 
- In Hall C we will install a new focusing spectrometer system, called the Super High Momentum Spectrometer (SHMS) with central momentum up to $11 \mathrm{GeV} / \mathrm{c}$ will enable measurements of particles scattered at up to full beam momentum. It will be used together with the existing High Momentum Spectrometer (HMS). Features of the SHMS include:

o High precision and reproducibility due to a rigid attachment to a central pivot and a robust support and transport structure;

o Ability to analyze charged particles up to the full 5-pass beam energy;

o Excellent momentum resolution for charged particles;

- Particle identification for charged particles via time-of-flight, electromagnetic shower calorimeters, and Cerenkov detectors;

- Flexibility to accommodate a variety of target types; and

o Capability to use luminosities of $1038 \mathrm{~cm}-2 \mathrm{~s}-1$.

In addition there is, of course, a great deal of civil construction to house the new GlueX experiment.

\section{Outline of the science to be explored at $12 \mathrm{GeV}$}

In the process of reaching CD- 1 , we have defined a clear set of physics priorities for the first 5 years of operation after the completion of construction [1, 2]. These priorities, which were judged to be outstanding, with significant discovery potential, by the independent DOE review [3] (in April 2005) will, of course, be subject to continual peer review and re-evaluation. However, there is no known or planned facility which can address those scientific challenges and it is difficult to imagine their importance diminishing in the intervening period.

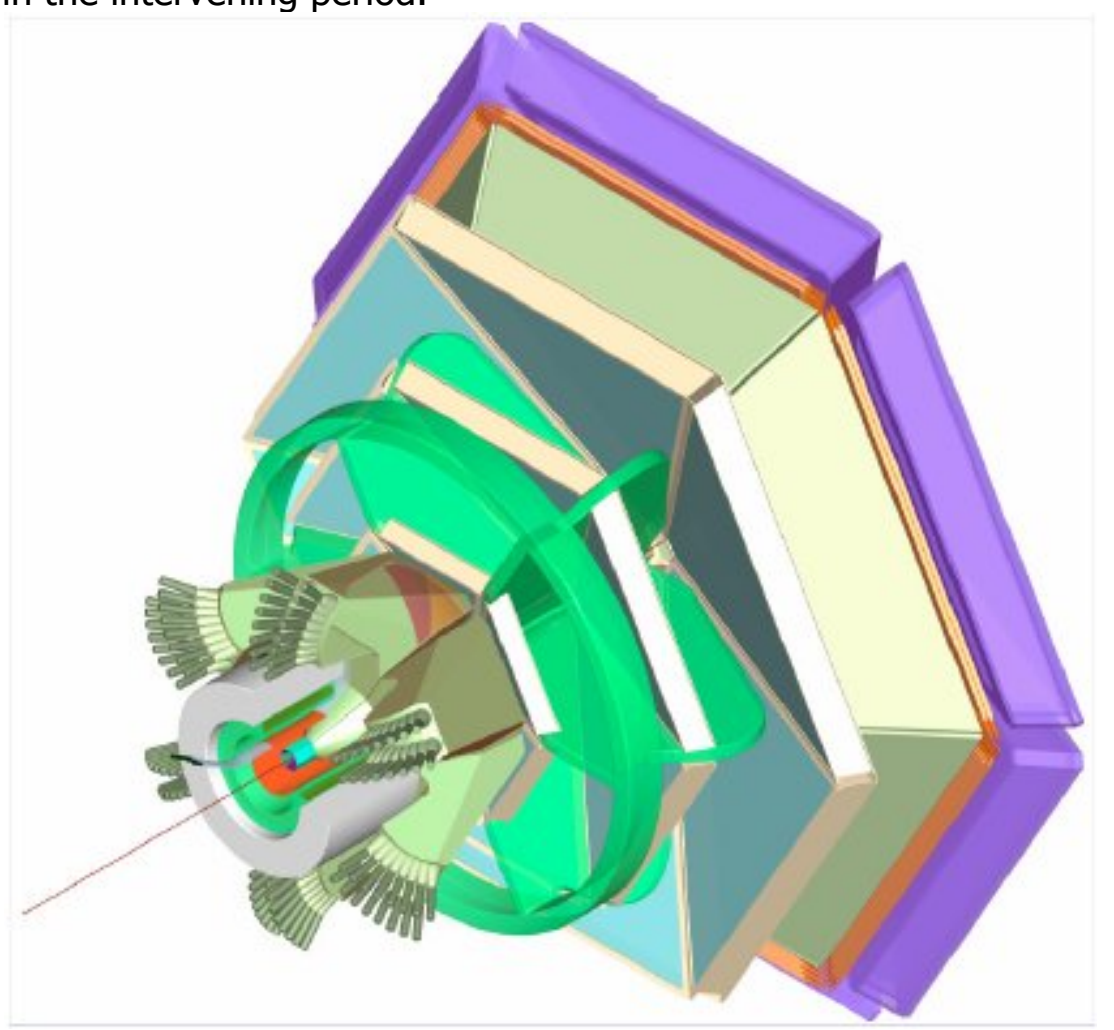

Fig. 3. The new CLAS 12 detector to be installed in Hall B. 


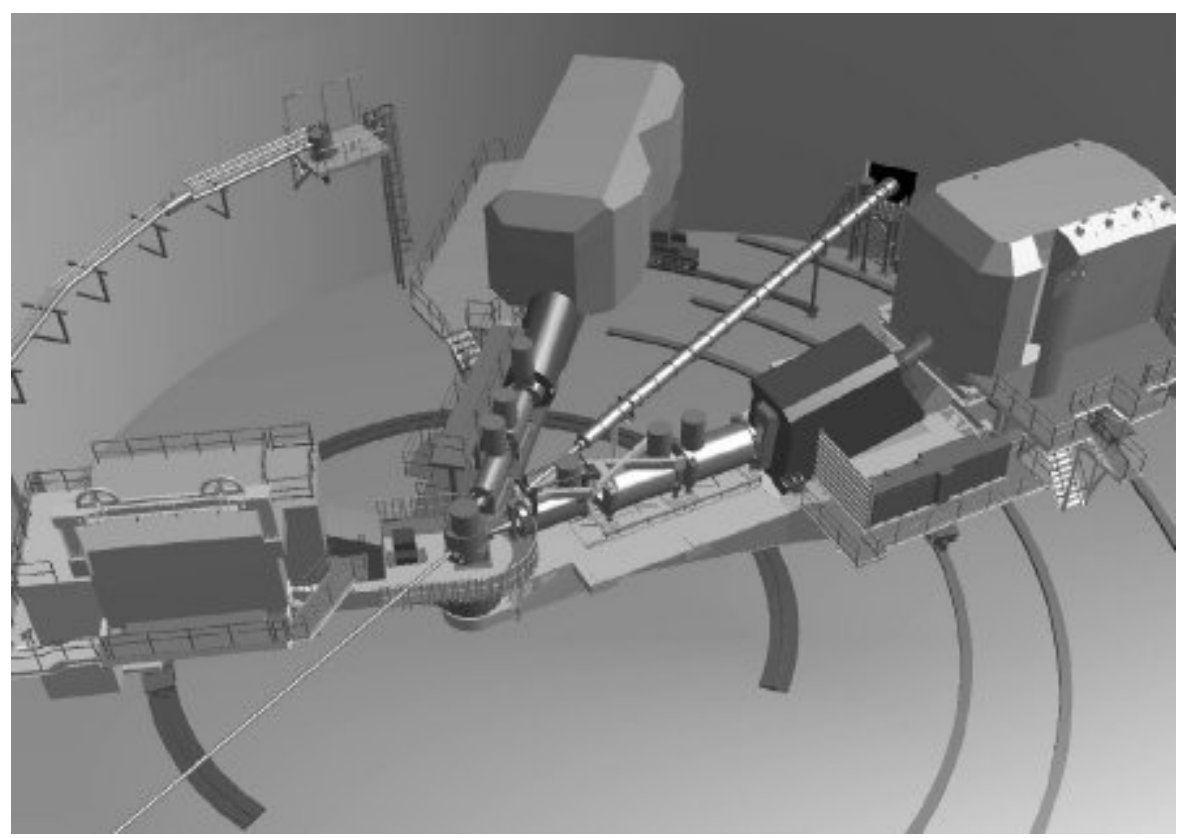

Fig. 4. The Super High Momentum Spectrometer (SHMS) planned for Hall C is shown together with the two existing spectrometers. The capability of either using SHMS alone, or in combination with the HMS, presents an extremely versatile and powerful set of tools for investigating deep hadron structure.

The major physics aims of the first 5 years of operation are:

- Revolutionize our knowledge of spin and flavor dependence of valence PDFs.

- Revolutionize our knowledge of the distribution of charge and current within the nucleon.

- Provide a totally new view of hadron (and nuclear) structure through the Generalized Parton Distributions (GPDs).

- Explore QCD in the nonperturbative regime. In particular, establish the existence and properties of exotic mesons and hence explore the mechanism of quark confinement.

- Establish a new paradigm for nuclear physics through the quest to understand nuclear structure in terms of QCD. In particular, the unique features of this new facility will enable us to explore the spin and flavor dependence of the famous EMC effect. We will also be able to study the propagation of quarks through nuclear matter.

- Make precision tests of the Standard Model.

We now briefly outline the main themes associated with each of these topics.

\subsection{Exotic mesons and the origin of confinement}

Unlike any area of physics hitherto explored, QCD has the property that the force carriers themselves, the gluons, can by themselves form new structures. In combination with quarkanti-quark pairs, gluons can also give rise to new particles whose quantum numbers cannot be made from a quark-anti-quark pair alone. The former are known as glueballs and the challenge to find them has been the lack of any distinct quantum 
numbers, at least in the low mass regime, and the fact that they almost certainly mix strongly with normal mesons. Exotic mesons, on the other hand, are expected to occur at low mass and, precisely because of the signature of their quantum numbers, be readily identifiable. Indeed, the best available theory suggests as many as three nonets of these "exotic mesons" in the mass range 1.5 to $2.5 \mathrm{GeV}$. The choice of $12 \mathrm{GeV}$ was precisely in order to make this mass region accessible with a proton target. The GlueX detector was specifically designed to have extremely high efficiency for detecting the multimeson final states resulting from the decay of these exotic mesons. Sophisticated techniques for making the partial wave analysis (PWA) needed to recognize an exotic meson signal have been underway at Indiana University for some years, with recent support at Jefferson Lab.

The physics interest in these states arises because the unique quantum numbers have their origin in excitations of the flux tube or string joining the quark-anti-quark pair. This flux tube is intimately connected with the nature of the QCD vacuum and the origin of confinement itself. Establishing the existence and properties of these exotics is a crucial step to understanding whether QCD really is the full theory of the strong interaction.

\subsection{Spin and flavor dependence of PDFs}

It is an astonishing fact that more than 30 years after the discovery of scaling we still do not know the distribution of the momentum of the proton on valence down quarks [4, 5]. For Bjorken $x$ beyond 0.6 , the uncertainty in $d(x)$ rapidly grows to $100 \%$. It is crucial to measure the large $x$ behavior of $d(x)$ in order to understand the relative importance of short and long distance di-quark correlations in the valence structure of the proton. It is at least equally important to understand how the spin of the proton is carried by its constituents [6]. With the $12 \mathrm{GeV}$ Upgrade Jefferson Lab will be able to unambiguously map out the distribution of momentum and spin on the valence $u$ and $d$ quarks in the proton. It will also be possible to explore the sea down to Bjorken $\mathrm{x}$ of order 0.1 .

\subsection{Distribution of charge and current in the nucleon}

Over the past 8 years, Jefferson Lab has mapped the electromagnetic form factors of the proton and neutron with great precision, out to a momentum transfer of order 4 GeV2 [6]. The $12 \mathrm{GeV}$ Upgrade will permit the exploration to momentum transfers of order $14 \mathrm{GeV} 2$, an increase in spatial resolution by a factor of two.

\subsection{Generalized parton distributions}

The GPDs will yield unique, tomographic information on the three-dimensional structure of nucleons and nuclei $[8,9]$ with particular sensitivity to the orbital angular momentum carried by specific quark flavors [10].

\subsection{The QCD basis of nuclear structure}

In more than 20 years since the discovery of the EMC effect (European Muon Collaboration) [11], which showed a dramatic change of the parton distribution functions of nuclei compared with free nucleons, no consensus has been reached as to the underlying physical origin of the effect [12]. It seems clear, however, that standard nuclear binding and kinematic corrections cannot explain the data and one is actually exploring the change in structure of the nucleonlike clusters when imbedded in the nuclear medium [13-15]. These are extremely important questions, going to the heart 
of how QCD itself yields nuclear matter $[16,17]$. It is quite clear that precise data exploring the flavor and spin dependence of this effect $[13,14]$ will be crucial in choosing amongst the various theoretical models so far proposed to explain it. There will also be a possibility to explore the interaction of fast quarks with nuclear matter, yielding new insights into the process of hadronisation, the nature of confinement and the change of the structure of the QCD vacuum in-medium.

\subsection{Beyond the standard model}

Precise measurements of parity violation, in Moller scattering and in hadronic deep inelastic scattering, as well as in atomic physics, have the potential to test for deviations from the predictions of the Standard Model. In this way one can in fact be sensitive to new physics, such as supersymmetry, additional gauge bosons and so on, at energy scales far beyond what can be explored directly at high energy facilities. A number of experiments are under consideration and the choice will be made on the highest impact possible with the beam characteristics provided through the Upgrade.

This is a rich program of great breadth and importance for our field. Much of what has been outlined here will be achieved within the first 5 years of operation. We remain completely open to new ideas from anyone, with access to the facility determined by an independent Program Advisory Committee on the basis of scientific feasibility and impact. With an active and growing user base of more than 1,000 scientists we can be certain that many of the best ideas are yet to come!

\section{Extracting hadron properties from lattice QCD}

It will be quite a few years before full QCD simulations can be performed at physical light quark masses. With computation time scaling like 1/m^3.6_q [18], quark masses are typically restricted to the range above $50 \mathrm{MeV}$ for accurate calculations with improved quark and gluon actions. The exception to this is to use staggered fermions which have shown some results at $25 \mathrm{MeV}$ [19] - but this approach has a number of technical problems, including a larger effective lattice spacing for fermions and multiple pions (extra tastes). The latter, especially, complicates the process of chiral extrapolation - the process of extrapolating hadron properties as a function of quark or pion mass to the physical point. In this chapter we will explain the concept of finite range regularization, which has proven so successful in permitting accurate chiral extrapolation in those cases where we know the chiral coefficients.

\subsection{Finite range regularization}

The power of effective field theory for strongly interacting systems has been apparent for more than 50 years, beginning with Foldy's inclusion of the anomalous magnetic moment of the proton in a derivative expansion of the electromagnetic coupling of photons to baryons. Of course, the fact that nucleon form factors can be approximated by a dipole with mass parameter $\mathrm{M} 2=0.71 \mathrm{GeV} 2$ means that the radius of convergence of the formal expansion is less than $0.84 \mathrm{GeV}$. In the last twenty years our understanding of the symmetries of QCD have led to a systematic expansion of hadron properties and scattering amplitudes about the limits $\mathrm{m} n=0$ and $\mathrm{q}=0$, known as chiral perturbation theory. For the pseudoscalar mesons, which are rigorously massless in the limit where the $u, d$ and s quark masses are zero (the chiral SU(3) limit), this approach has been especially successful. Although, even here, serious questions have been raised 
over whether the formal expansion is really convergent for masses as large as ms [2022].

We begin our discussion of chiral effective field theory by briefly recalling the expansion of the nucleon mass as a function of $\mathrm{m} n$, a case which has been widely studied and where there is no question concerning the chiral coefficients. The formal chiral expansion for $\mathrm{MN}$, in terms of $\mathrm{mn}$, about the $\mathrm{SU}(2)$ chiral limit in full QCD is:

$$
\begin{aligned}
M_{N}= & a_{0}+a_{2} m_{\pi}^{2}+a_{4} m_{\pi}^{4}+a_{6} m_{\pi}^{6}+\ldots \\
& +\sigma_{\mathrm{N}_{\pi}}\left(m_{\pi}\right)+\sigma_{\Delta \pi}\left(m_{\pi}\right),
\end{aligned}
$$

where $\sigma_{\mathrm{B}}$ is the self-energy arising from a $\mathrm{B} \Pi$ loop (with the dominant terms involving $\mathrm{B}$ $=\mathrm{N}$ or $\Delta$ ). These $\mathrm{N}$ and $\Delta$ loops generate the leading and next-to-leading non-analytic (LNA and NLNA) behaviour, respectively.

These loop integrals contain ultraviolet divergences which require some regularisation prescription. The standard approach is to use dimensional regularisation to evaluate the self-energy integrals. Under such a scheme the $\mathrm{NN} n$ contribution simply becomes $\sigma_{\mathrm{Nn}}\left(\mathrm{m}_{\Pi}\right) \rightarrow \mathrm{C}_{\mathrm{LNA}} \mathrm{m}_{\Pi}^{3}$ and the analytic terms, $\mathrm{a}_{0}$ and $\mathrm{a}_{2} \mathrm{~m}_{\mathrm{n}}^{2}$, undergo an infinite renormalisation. The $\Delta$ contribution

produces a logarithm, so that the complete series expansion of the nucleon mass about $\mathrm{m}_{\Pi}=0$ is:

$$
M_{N}=c_{0}+c_{2} m_{\pi}^{2}+c_{4} m_{\pi}^{4}+c_{\mathrm{LNA}} m_{\pi}^{3}+c_{\mathrm{NLNA}} m_{\pi}^{4} \log m_{\pi}+\ldots,
$$

where the $a_{i}$ have been replaced by the renormalised (and finite) parameters $c_{i}$.

The coefficients of the low-order, non-analytic contributions are known [25-27]:

$$
c_{\mathrm{LNA}}=-\frac{3}{32 \pi f_{\pi}^{2}} g_{A}^{2}, \quad c_{\mathrm{NLNA}}=\frac{3}{32 \pi f_{\pi}^{2}} \frac{32}{25} g_{A}^{2} \frac{3}{4 \pi \Delta} .
$$

Although strictly one should use values in the chiral limit, we take the experimental numbers with $\mathrm{g}_{\mathrm{A}}=1.26, \mathrm{f}_{\mathrm{n}}=0.093 \mathrm{GeV}$, the $\mathrm{N}-\Delta$ mass splitting, $\Delta=0.292 \mathrm{GeV}$ and the mass scale associated with the logarithms will be taken to be $1 \mathrm{GeV}$.

We stress that Eq. (2) was derived in the limit $m_{\Pi} / \Delta<<1$. At just twice the physical pion mass this ratio approaches unity. Mathematically the region $\mathrm{m}_{\Pi} \approx \Delta$ is dominated by a square root branch cut which starts at $m_{\Pi}=\Delta$. Using dimensional regularisation this takes the form [28]:

$$
\frac{-3}{32 \pi f_{\pi}^{2}} \frac{32}{25} g_{A}^{2} \frac{1}{2 \pi}\left[\left(2 \Delta^{3}-3 m_{\pi}^{2} \Delta\right) \log \left(\frac{m_{\pi}^{2}}{\mu^{2}}\right)-2\left(\Delta^{2}-m_{\pi}^{2}\right)^{\frac{3}{2}} \log \left(\frac{\Delta-\sqrt{\Delta^{2}-m_{\pi}^{2}}}{\Delta+\sqrt{\Delta^{2}-m_{\pi}^{2}}}\right)\right]
$$

for $m_{\Pi}<\Delta$, while for $m_{\Pi}>\Delta$ the second logarithm becomes an arctangent. Clearly, to access the higher quark masses in the chiral expansion, currently of most relevance to lattice QCD, one requires a more sophisticated expression than that given by Eq. (2).

In order to concentrate on the essentials of the convergence problem, we initially ignore the $\Delta_{\Pi}$ cut. We note that the formal expansion of the $\mathrm{N} \rightarrow \mathrm{N} \Pi \rightarrow \mathrm{N}$ self-energy integral, $\sigma_{\mathrm{N} n}$, has been shown to have poor convergence properties. Using a sharp, ultra-violet cut-off, Wright showed [29] that the series expansion, truncated at $\mathrm{O}\left(\mathrm{m}_{n}^{4}\right)$, diverged for $\mathrm{m}_{\Pi}>0.4 \mathrm{GeV}$. This already indicated that the series expansion motivated by dimensional regularisation would have a slow rate of convergence. A similar conclusion had been 
reached somewhat earlier by Stuckey and Birse [23]. Figure 5 graphically illustrates the lack of convergence for $\mathrm{m} n$ beyond about $0.3 \mathrm{GeV}$ for the nucleon mass (with the coefficients determined by the procedure explained below). A similar result has been found for the proton magnetic moment [43].

In considering the convergence of the truncated series, Eq. (2), it is helpful to return to the general form from which it was derived, namely Eq. (1). The dimensionally regulated approach requires that the pion mass should remain much lighter than every other mass scale involved in the problem. This requires that $m_{\pi} / \Lambda_{\mathrm{XSB}}<<1$ (and $\mathrm{m}_{\pi} / \Delta<<1$ if we use the simple logarithm in Eq.(2) rather than the full cut structure of Eq.(4)).

An additional mass scale, which we address in detail below, is set by the physical extent of the source of the pion field [30]. This scale, which is of order $\mathrm{R}_{\text {SOURCE, }}^{-1}$ corresponds to the transition between the rapid, non-linear variation required by chiral symmetry and the smooth, constituent-quark like mass behaviour observed in lattice simulations at larger quark mass. An alternative to dimensional regularisation is to regulate Eq. (1) with a finite ultra-violet cut-off in momentum space.

In order to put these observations in a proper context we now outline the key features of the pedagogic introduction to effective field theories given a few years ago by LePage [24]. In that case the problem considered was to develop an effective field theory for the solution of the Schrödinger equation for an unknown potential. The key physical idea of the effective field theory is to introduce an energy scale, $\lambda$, above which one does not attempt to understand the physics. For example, the energy region above that cut-off may involve new physics - e.g., in the very high energy limit, physics beyond the Standard Model. Because one does not pretend to control physics above the scale $\lambda$, one should not include momenta above $\lambda$

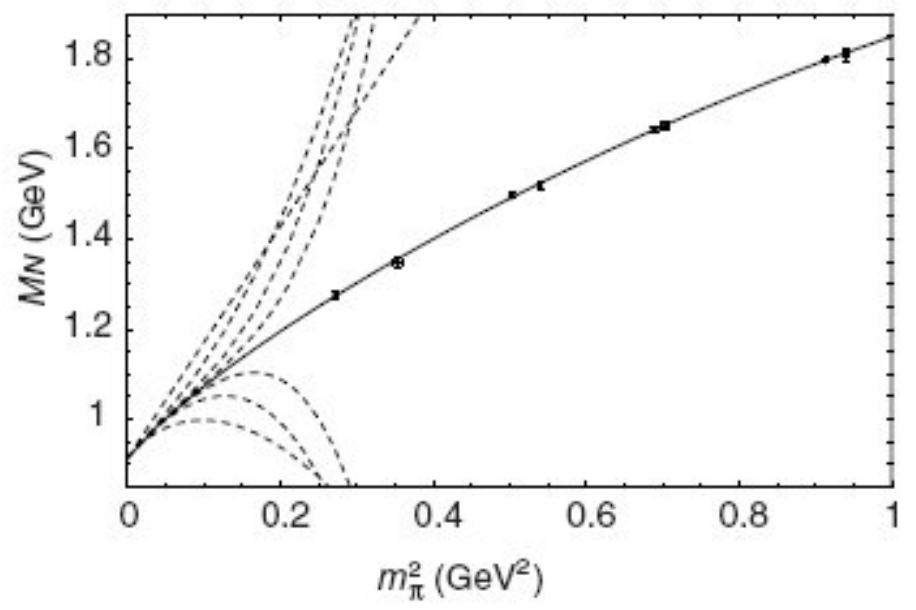

Fig. 5. Fits to lattice data with a dipole regulator for the self-energy loop $\mathrm{N} \rightarrow \mathrm{N}_{\Pi}$ (neglecting the $\mathrm{N} \rightarrow \Delta_{\Pi}$ term for simplicity). The dashed lines show the expansion up to successive orders in $\mathrm{m}_{\pi,}$ from $\mathrm{m}_{n}^{2}$ through $\mathrm{m}_{n}^{8}$ - these alternate about the solid curve as we stop at an even or odd number of terms.

in computing radiative corrections. Instead, one introduces renormalisation constants which depend on (or "run with") the choice of cut-off $\lambda$ so that, to the order one works, physical results are independent of $\lambda$. 
As LePage points out, "it makes little sense to reduce a [i.e., increase $\lambda \sim 1$ /a] below the range" in which we understand the physics, because the "structure they see there is almost certainly wrong." When it comes to specific problems with the development of an effective theory of NN scattering, this line of argument leads him to conclude that "the problem is with dimensional regularization - and not with effective field theory." In particular, dimensional regularization involves integrating loop momenta in the effective field theory over momenta all the way to infinity - way beyond the scale where the effective theory has any physical significance.

In the context of our present problem of chiral extrapolation, the essential lesson to draw from LePage's investigation is that one should not take the cut-off to $\infty$, that is one should not use dimensional regularization. Nor should one attempt to determine the "true" cut-off of the theory. Rather, one should choose a cut-off scale somewhat below the place where the effective theory omits essential physics and use data to constrain the renormalisation constants of the theory and hence eliminate the dependence on that cut-off as far as possible.

The issue is then what mass scale determines the upper limit beyond which the effective field theory is applicable. This is commonly taken to be $\Lambda_{X P T} \sim 4 \pi f_{\pi} \sim 1 \mathrm{GeV}$. Unfortunately this is incorrect for baryons. In the context of nuclear physics it has long been appreciated that nuclear sizes could never be derived from naive dispersion relation considerations of nearest t-channel poles - anomalous thresholds associated with the internal structure of nuclei dominate. Similarly, the size of a baryon is determined by nonperturbative QCD beyond the scope of chiral perturbation theory. The natural scale associated with the size of a nucleon is the inverse of its radius or a mass scale $\mathrm{R}_{\text {SOURCE }}^{-1} \sim 0.2$ to $0.5 \mathrm{GeV}-$ far below $\Lambda_{X P T}$. In the context of effective field theory it is inconsistent to keep loop contributions from momenta above this scale!

As a result of these considerations, we are led to regulate the radiative corrections which give rise to the leading and next-to-leading non-analytic contributions to the mass of the nucleon using a finite range regulator with a mass in the range $R_{\text {SOURCE }}^{-1}$. As far as possible residual dependence on the specific choice of mass scale (and form of regulator function) will be eliminated by fitting the renormalisation constants to nonperturbative QCD - in this case data obtained from lattice QCD simulations. The quantitative success of applying the method is to be judged by the extent to which physical results extracted from the analysis are indeed independent of the regulator (over a physically sensible range). We refer to this approach as finite range regularization, FRR.

\subsection{Numerical results for the nucleon}

Having established the philosophical framework for our analysis we now investigate the effective rate of convergence of the chiral expansions obtained using different functional forms for the regulator. We also make comparisons with the results obtained using a dimensionally regulated approach. To analyse the merits of various regularisation schemes, at best, we would require exact knowledge of how the nucleon mass varies with quark mass. Having only one value for the experimental nucleon mass we cannot determine the parameters that govern the quark mass dependence of $M_{N}$ without taking some information from alternate sources. 
Lattice QCD gives us a reliable, non-perturbative method for studying the variation of $\mathrm{M}_{N}$ with $\mathrm{m}_{\pi}$. In principle, this allows one to fix the parameters of the chiral expansion using data obtained in simulations performed at varying quark mass. Lattice simulations of full QCD are restricted to the use of relatively heavy quarks and hence it is not clear, a priori, whether the effective field theory expansion is capable of linking to even the lightest simulated quark mass where $\mathrm{m}_{\pi} \sim 500 \mathrm{MeV}$. As input we use both the physical nucleon mass and recent lattice QCD results of the CP-PACS Collaboration [31] and the JLQCD Collaboration [32]. This enables us to constrain an expression for $M_{N}$ as a function of the quark mass.

The numerical results of Ref. [31] have been obtained using improved gluon and quark actions on fine, large volume lattices with high statistics. These simulations were performed using an Iwasaki gluon action [33] and the mean-field improved clover fermion action. In this work we concentrate on only those results with $\mathrm{m}_{\text {sea }}=\mathrm{m}_{\mathrm{va}} \mathrm{l}$ and the two largest values of $\beta$ (i.e., the finest lattice spacings a $\sim 0.09-0.13 \mathrm{fm}$ ). We use just the largest volume results of Ref. [32], where simulations were performed with nonperturbatively improved Wilson quark action and plaquette gauge action. The lattice volumes and spacings are similar for the two data sets.

We set the physical scale at each quark mass, using the UKQCD method [34]. That is, we use the Sommer scale $r_{0}=0.5 \mathrm{fm}[35,36]$. This choice is ideal in the present context because the static quark potential is insensitive to chiral physics. This ensures that the results obtained represent accurate estimates of the continuum, infinite-volume theory at the simulated quark masses. The lattice data lies in the intermediate mass region, with $\mathrm{m}_{n}^{2}$ between 0.3 and $1.0 \mathrm{GeV}^{2}$.

To remove the bias implicit in choosing any particular regularization scheme, we allow each scheme to serve as a constraint curve for the other methods. In this way we generate six (one for each regularisation) different constraint curves that describe the quark mass dependence of $\mathrm{M}_{N}$. The first case corresponds to the truncated power series obtained through the dimensionally regulated (DR) approach, Eq. (2). We work to analytic order $\mathrm{m}_{\pi}^{6}$, which is necessary in order to remove incorrect short distance physics arising from the non-analytic contribution at order $\mathrm{m}^{4}{ }_{n} \log \mathrm{m}_{\pi}$. The second procedure (labeled "BP") takes a similar form but the branch point obtained from the dimensionally regularized $\mathrm{N} \rightarrow \Delta \Pi$ transition is retained in its full functional form. That is, the logarithm in Eq. (2) is replaced by the full expression, Eq. (4), which ensures the correct non-analytic structure where the logarithm converts to an arctangent above the branch point. We refer to this form as the dimensionally-regulated branch-point (BP) approach.

The loop integrals can be written as $\sigma \mathrm{N}_{\Pi} \equiv \mathrm{c}_{L N A} \mathrm{I}_{\Gamma}\left(\mathrm{m}_{\Pi \prime} \Lambda\right)$ and $\sigma_{\Delta \Pi} \equiv \mathrm{c}_{N L N A} \mathrm{I}_{\Pi \Delta}\left(\mathrm{m}_{\Pi \prime}, \Lambda\right)$, where:

$$
I_{\pi}=\frac{2}{\pi} \int_{0}^{\infty} d k \frac{k^{4} u^{2}(k)}{k^{2}+m^{2}}
$$

and

$$
I_{\pi \Delta}=-\frac{8 \Delta}{3} \int_{0}^{\infty} d k \frac{k^{4} u^{2}(k)}{\sqrt{k^{2}+m^{2}\left(\Delta+\sqrt{k^{2}+m^{2}}\right)}} .
$$

As a test of model dependence, we employ four different functional forms for the finiteranged, ultra-violet vertex regulator, $\mathrm{u}(\mathrm{k})$ - namely the sharp-cutoff $(\mathrm{SC}), \theta(\Lambda-\mathrm{k})$; 
monopole (MON), $\Lambda^{2} /\left(\Lambda^{2}+k^{2}\right)$; dipole (DIP), $\Lambda^{4} /\left(\Lambda^{2}+k^{2}\right)^{2}$; and Gaussian (GAU), $\exp \left(-k^{2} / \Lambda^{2}\right)$. Closed expressions for these integrals in the first three cases are given in the Appendix of Ref. [37].

Provided one regulates the effective field theory below the point where new short distance physics becomes important, the essential results will not depend on the cut-off scale [24]. We use Ref. [37] as a guide for the appropriate scales for each of the regulator forms. In particular

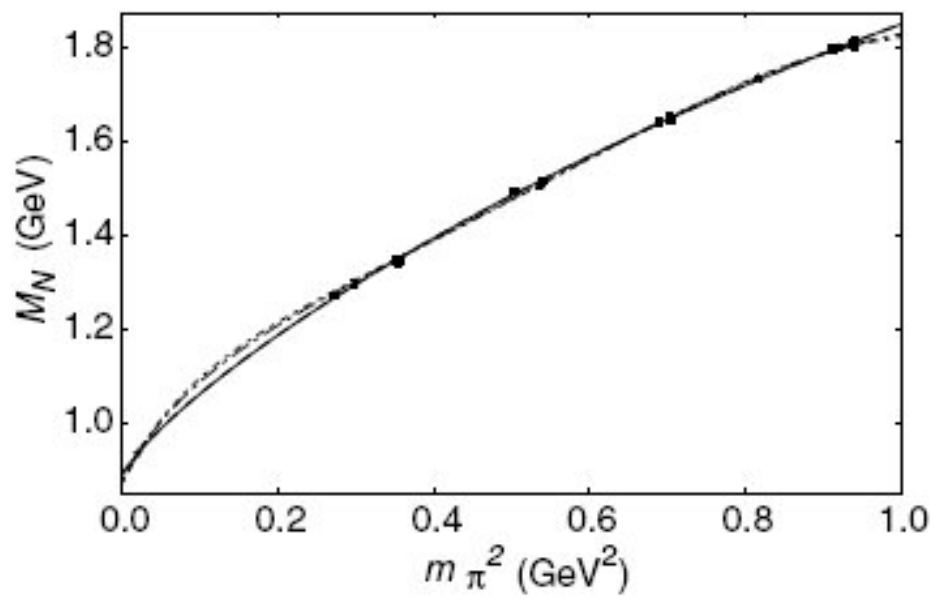

Fig. 6. Constraint curves for the variation of $\mathrm{MN}$ with pion mass given by the various regularization schemes. The short dash curve corresponds to the simple dimensional regularisation scheme (DR) and the long dash curve to the more sophisticated dimensionally regulated approach (BP). The four finite range regulators (solid curves) are indistinguishable at this scale. Oscillations of the dimensionally regulated schemes (dashed curves) about the solid lines are apparent. This is typical for a low-order polynomial fit.

Table 1. The bare fit parameters obtained for the constraint curves, $M\left(m_{n}\right)$ (in appropriate powers of $\mathrm{GeV}$ ), using the regularization schemes explained in the text.

\begin{tabular}{lllcc}
\hline \hline Regulator & \multicolumn{1}{c}{$a_{0}$} & $a_{2}$ & $a_{4}$ & $a_{6}$ \\
\hline DR & 0.877 & 4.06 & 5.57 & -3.24 \\
BP & 0.821 & 4.57 & 8.92 & -2.02 \\
SC & 1.02 & 1.15 & -0.359 & 0.055 \\
MON & 1.58 & 0.850 & -0.162 & -0.007 \\
DIP & 1.22 & 0.925 & -0.177 & -0.009 \\
GAU & 1.11 & 1.03 & -0.265 & 0.024 \\
\hline \hline
\end{tabular}

we choose regulator masses of $0.4,0.5,0.8$ and $0.6 \mathrm{GeV}$ for the sharp, monopole, dipole and Gaussian regulators, respectively. For all six regularisation prescriptions, both DRbased and FRR, we fit four free parameters to constrain the expansion of the nucleon mass. With the values of the non-analytic contributions fixed to their model-independent values, the coefficients of analytic terms up to $\mathrm{m}^{6}{ }_{n}$ are allowed to vary to best fit the lattice simulation results and to reproduce the physical nucleon mass. The resultant curves are displayed in Fig. 6. To the naked eye all of the curves are very much in agreement with each other. All of them are able to give an accurate description of the lattice data and match the physical value of $\mathrm{M}_{N}$. 
The best fit parameters for our constraint curves, $M\left(m_{n}\right)$, are shown in Table 1. We stress that the parameters listed in this table are bare quantities and hence renormalisation scheme dependent. If one is to rigorously compare the parameters of the effective field theory, the self-energy contributions need to be Taylor expanded about $\mathrm{m}_{n}=0$ in order to yield the renormalisation for each of the coefficients in the quark mass expansion about the chiral limit. A comparison of the resulting quark-mass expansion for each of the regularisation schemes is shown in Table 2. The most remarkable feature of Table 2 is the very close agreement between the values of the renormalised coefficients, especially for the finite-range regularisation (FRR) schemes. For example, whereas the variation in $\mathrm{a}_{0}$ between all four FRR schemes is $50 \%$, the variation in $\mathrm{c}_{0}$ is a fraction of a percent. For $\mathrm{a}_{2}$ the corresponding figure is $30 \%$ compared with less than $9 \%$ variation in $\mathrm{c}_{2}$. If one excludes the sharp cut-off (SC) regulator, the monopole, dipole and Gaussian results for c2 vary by only $2 \%$. Finally, for c4 the agreement is good for the latter three schemes.

Table 2. Renormalised chiral expansion parameters for the constraint curves, $M\left(m_{n}\right)$ (all quantities are given in appropriate powers of $\mathrm{GeV}$ ). The regularisation schemes are explained in the text.

\begin{tabular}{lccc}
\hline \hline Regulator & $c_{0}$ & $c_{2}$ & $c_{4}$ \\
\hline DR & 0.877 & 4.10 & 5.57 \\
BP & 0.881 & 3.84 & 7.70 \\
SC & 0.895 & 3.02 & 14.0 \\
MON & 0.898 & 2.78 & 23.5 \\
DIP & 0.897 & 2.83 & 21.7 \\
GAU & 0.897 & 2.83 & 21.2 \\
\hline \hline
\end{tabular}

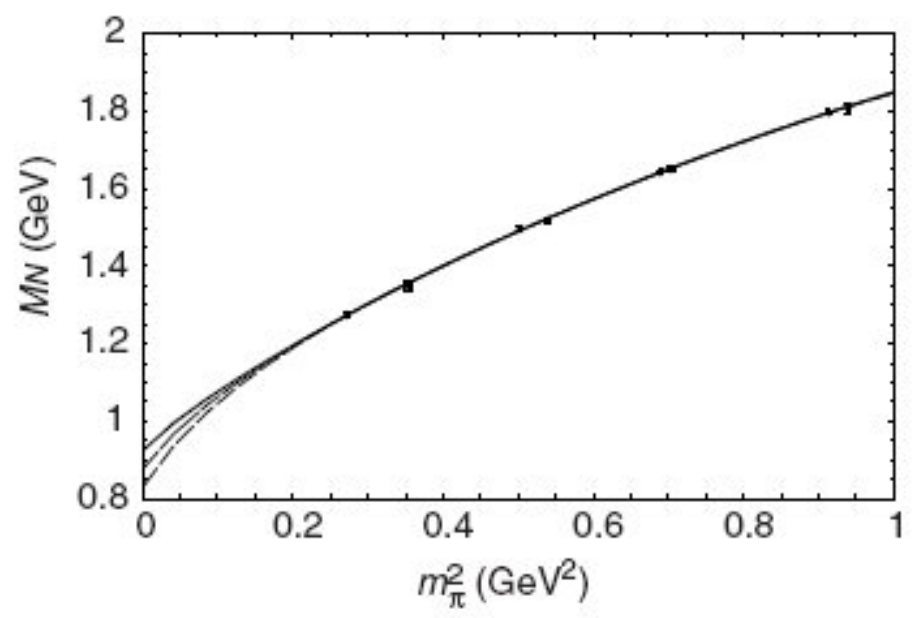

Fig. 7. Extrapolation of nucleon mass lattice data based on different regularisation schemes to order $\mathrm{m}_{q \text {. }}^{2}$ The four, indistinguishable, solid lines correspond to the finiterange regulators, the dashed curve is the dimensionally regularized fit. The dash-dot curve displays the DR form with the correct branch structure at $\mathrm{m}_{n}=\Delta$.

A comparison between $\mathrm{a}_{4}$ and $\mathrm{c}_{4}$ is especially important in order to understand why the FRR schemes are so efficient. Whereas the renormalised coefficients are consistently very large for the three smooth FRR schemes, the bare coefficients of the residual 
expansion are of order 100 times smaller! That is, once one incorporates the effect of the finite size of the nucleon by smoothly suppressing pion loops for pion masses above 0.4-0.5 GeV, the residual series expansion has vastly improved convergence properties.

In contrast, in order to fit the nucleon mass data over an extended range of pion mass, the dimensional regularisation schemes require bare expansion coefficients which are much larger, 30 times larger in the case of $\mathrm{a}_{4}$. Still these coefficients are not large enough to reach the consistent values of the smooth FRR results reported in Table 2.

The next challenge is, of course, to apply the FRR method of chiral extrapolation to the lattice data for the nucleon, without using the physical nucleon mass as a constraint [38] - i.e., to see what predictive power it has. In addition to the $N \Pi$ and $\Delta \Pi$ loops described earlier, we also include the so-called tadpole term, which also enters at NLNA [39]. The fits to the lattice data using all four FRR, as well as the two dimensionally regulated fits, are shown in Fig. 7. The FRR results display excellent mutual agreement. The dimensional regularization forms, particularly the branch-point form, show significantly improved fits over the leading order result.

In Table 3 we display the extrapolated nucleon mass for the various fits. We see remarkable agreement between the different orders of the expansion for the FRR fits. This ensures that the formal expansion associated with FRR effective field theory is sufficiently convergent. The NLNA dimensionally regularized forms are consistent with those of FRR within statistical error, yet do not offer that same level of stability.

Table 3. Extrapolated nucleon mass evaluated at the physical pion mass. The two columns describe extrapolations based on the LNA and NLNA forms (including the tadpole term in the latter case).

\begin{tabular}{lcc}
\hline Regulator & $M_{N}^{\text {(LNA) }}$ & $M_{N}^{\text {(NLNA) }}$ \\
\hline Dim. Reg. & 0.784 & 0.884 \\
Dim. Reg. (BP) & $\ldots$ & 0.923 \\
Sharp cutoff & 0.968 & 0.961 \\
Monopole & 0.964 & 0.960 \\
Dipole & 0.963 & 0.959 \\
Guassian & 0.966 & 0.960 \\
\hline
\end{tabular}

\subsection{The $\rho$ meson}

The CP-PACS Collaboration also presented a very thorough study of the $\rho$ meson at a variety of lattice spacings in both full and partially quenched QCD (PQQCD). Our study of this data has unexpectedly shown just how important PQQCD simulations can be [40]. A tremendous formal advantage of pQQCD is that the chiral coefficients entering there are precisely the same as those appearing in full QCD (alias the real world). Since the major time consuming step in lattice QCD is the construction of suitable gauge configurations, it is very cost effective to compute hadron properties for a variety of valence quark masses using the same sea quark masses.

Indeed, in the case of the $\rho$ meson the CP-PACS Collaboration [31] used mean-field improved clover fermions at four different couplings, $\beta$. For each value of the coupling, four different sea quark masses were calculated, yielding a total of 16 independent 
gauge field ensembles. On each ensemble, the quark propagator has been evaluated for five values of the valence quark mass. For the vector mesons constructed of degenerate valence quarks there are a total of 80 "data" points. This five-fold increase in the data sample, which can be used in the fitting process associated with chiral extrapolation, has the effect of dramatically improving the precision with which the final, physical $\rho$ meson mass can be determined. The lattice scale is set via the QCD Sommer scale $r_{0}=0.49 \mathrm{fm}$ [35], enabling all of these points to be shown in physical units - as in Fig. 8.

In the analysis the mass of a particular vector meson state is therefore described by

$$
M_{i j k}^{a}=M\left(a, m_{i} ; m_{j}, m_{k}\right),
$$

where the first two parameters of $M\left(a, m_{i}, m_{j r} m_{k}\right)$ specify the gauge field ensemble with lattice spacing a and sea-quark mass $m_{\text {sea }}=m_{i}$. The masses of the valence quarks are given by $\mathrm{m}_{j}$ and $\mathrm{m}_{k}$. Similarly, ${ }^{M_{i j k}}$ is the mass of a pseudoscalar meson of equivalent quark composition. The global parametrisation of the vector meson mass, dependent on quark masses, lattice spacing and physical volume, is written as

$$
M_{i j j}^{a}{ }^{2}=\left(\alpha_{0}+X_{2} a^{2}+\alpha_{2} m_{i j j}^{a}{ }^{2}+\alpha_{4} m_{i j j}^{a}{ }^{4}\right)^{2}+\Sigma_{a ; i j j}^{\mathrm{TOT}}(L) .
$$

The total loop correction to the ${ }^{M_{i j j}^{a}}$ meson, on a finite box of physical length $\mathrm{L}=\mathrm{Na}$, is described by

$$
\Sigma_{a ; i j j}^{\mathrm{TOT}}(L)=\Sigma_{\pi \pi}^{\rho}\left(m_{i i j}^{a}, L\right)+\Sigma_{\pi \omega}^{\rho}\left(m_{i i j}^{a}, L\right)+\Sigma_{\eta^{\prime} \rho}^{\rho}\left(m_{i i j}^{a}, m_{i j j}^{a}, m_{i i i}^{a}, L\right) .
$$

The relevant loop integrals, in the $\mathrm{L} \rightarrow \infty$ limit, are given by

$$
\Sigma_{\pi \pi}^{\rho}\left(m_{i i j}^{a}\right)=-\frac{f_{\rho \pi \pi}^{2}}{24 \pi^{3}} \int d^{3} k \frac{k^{2} u_{\pi \pi}^{2}(k)}{\sqrt{k^{2}+m_{i i j}^{a}{ }^{2}}\left[k^{2}+m_{i i j}^{a}{ }^{2}-\mu_{\rho}^{2} / 4\right]},
$$

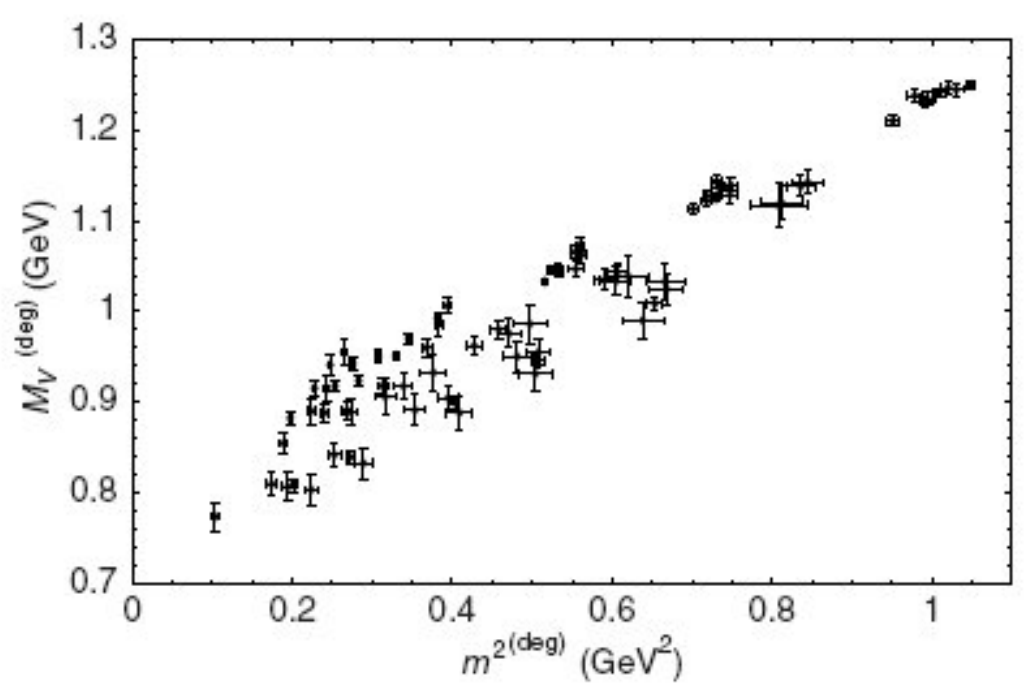

Fig. 8. Partially-quenched vector meson masses plotted versus "degenerate" pion mass squared. Here $\mathrm{M}_{\nu}^{(\mathrm{deg})}={ }^{M_{i j j}}$ and $\mathrm{m}_{n}^{(\mathrm{deg})}=m_{i j j}^{i}$. The simulation results are from CP-PACS [31]. 


$$
\begin{gathered}
\Sigma_{\pi \omega}^{\rho}\left(m_{i i j}^{a}\right)=-\frac{\mu_{\rho} g_{2}^{2}}{12 \pi^{3} f_{\pi}^{2}} \int d^{3} k \frac{k^{2} u^{2}(k)}{\sqrt{k^{2}+m_{i i j}^{a}{ }^{2}}\left[\sqrt{k^{2}+m_{i i j}^{a}{ }^{2}}+\left(M_{i i j}^{a}-M_{i j j}^{a}\right)\right.}, \\
\Sigma_{\eta^{\prime} \rho}^{\rho}\left(m_{i i j}^{a}, m_{i j j}^{a}, m_{i i i}^{a}\right)= \\
\frac{\mu_{\rho} g_{2}^{2}}{12 \pi^{3} f_{\pi}^{2}} \int d^{3} k \frac{k^{2} u^{2}(k)}{\left(k^{2}+m_{i j j}^{a}\right)}\left\{\frac{\left(m_{i i j}^{a}{ }^{2}-m_{i j j}^{a}{ }^{2}\right)}{\left(k^{2}+m_{i i j}^{a}{ }^{2}\right)}+\frac{\left(m_{i i i}^{a}{ }^{2}-m_{i j j}^{a}{ }^{2}\right)}{\left(k^{2}+m_{i j j}^{a}{ }^{2}\right)}\right\} .
\end{gathered}
$$

Here the physical $\rho$ and $n$ masses, $\mu_{\rho}=0.770 \mathrm{GeV}$ and $\mu_{n}=0.140 \mathrm{GeV}$; $\rho п \Pi$ coupling $\mathrm{f}_{\text {опп }}=6.028$ [41]; $\mathrm{g}_{2}$ (as introduced in Ref. [49]) is related to the $16 \mathrm{GeV}^{-1}[50]$, by $\mathrm{g}_{2}=\mathrm{g}_{\omega \text { onff }} / 2=0.74 ; \mathrm{k}=|\mathrm{k}|$; finite-range regularisation is implemented with a dipole form, $\mathrm{u}(\mathrm{k})=\left(1+\mathrm{k}^{2} / \Lambda^{2}\right)^{-2}$, and the $\rho п \Pi$ coupling is preserved at the physical threshold, $u_{\pi \pi}(k)=u(k) u^{-1}\left(\sqrt{\mu_{\rho}^{2} / 4-\mu_{\pi}^{2}}\right)$.

The leading finite-volume corrections are trivially incorporated by replacing the continuum loop integrals in Eqs. $(10,11,12)$ by a sum over discrete momenta [41-43]

$$
\int d^{3} k \rightarrow\left(\frac{2 \pi}{L}\right)^{3} \sum_{k}
$$

where $\mathrm{k}=(2 \pi / L)$ ifor $i \in Z 3$. This modification produces an infrared suppression of the loop integrals. It is also independent of the choice of ultra-violet regularization [45].

The bracketed term in Eq. (8) describes the residual variation of the vector meson mass which is not contained in the one-loop Goldstone boson diagrams. The analytic variation of the quark-mass dependence is characterised by the continuum parameters, ai. At finite lattice spacing, all terms at order $a$ and $a^{2}$ must be treated consistently with the symmetry breaking patterns of the prescribed fermion action [47]. This can potentially lead to more singular chiral behaviour in the effective field theory at finite lattice spacing [51]. In this study, the leading lattice spacing corrections to the terms analytic in the quark mass were included.

The form provided by Eq. (8) allows a universal fit to all these points with just four free parameters (plus regulator scale). This is therefore a highly constrained fit to the large sample of simulation results. The $X^{2}$ indicates that Eq. (8) accurately describes this large quantity of data. The regulator mass, $\Lambda$, was optimised to produce a best fit to the data, namely $\Lambda=655 \pm 35 \mathrm{MeV}$, with the bound determined statistically from the $1 \sigma$ variation about the central fit. Studying the variation of the fit over this domain introduces a small additional uncertainty to the extrapolated result which is listed below in the error estimate. 


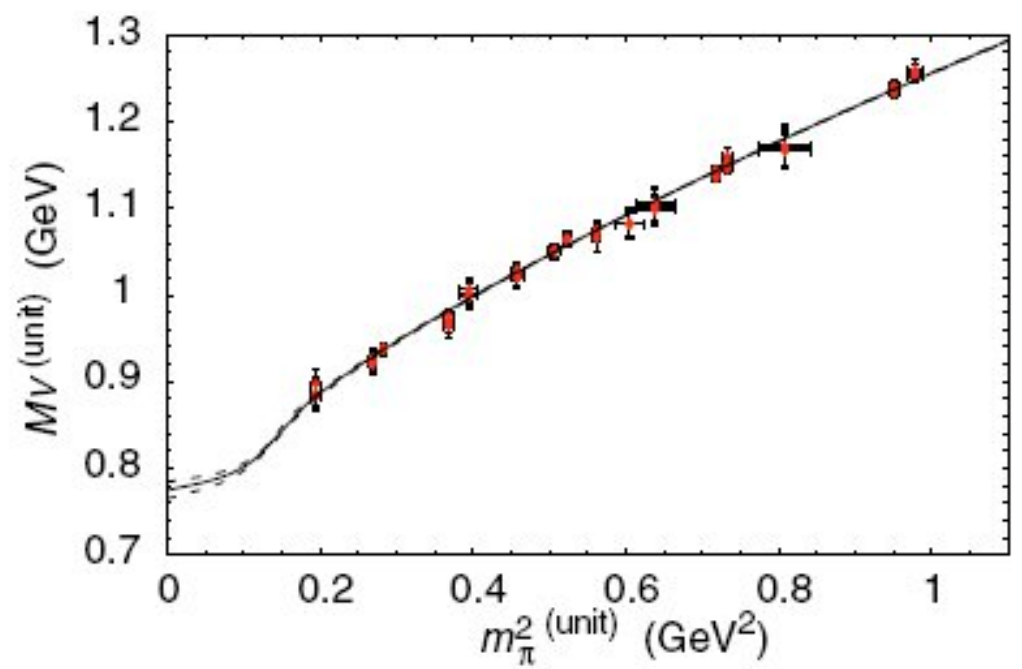

Fig. 9. The same 80 lattice data points as in Fig. 2, after correction to restore the infinite-volume, continuum and quark-mass unitarity limits. The central curve displays the best-fit from the global analysis. The dashed curves show the bounds on the FRR scale, $0.620<\Lambda<0.690 \mathrm{GeV}$.

Variations of Eq. (8) have also been investigated. Scaling corrections to the parameters $\mathrm{a}_{2}$ and $\mathrm{a}_{4}$ yield coefficients which are consistent with zero. Linear corrections in the lattice spacing, taking the form of a term $X_{I}$ a, are observed to be small and hence do not improve on the fit with $\mathrm{X}_{1}=0$. Extending to higher analytic order in the quark mass expansion, by a term $a_{6} m_{n,}{ }_{n}$, reduces the stability of the fit, indicating that the data are consistent with $\mathrm{a}_{6}=0$-see Ref. [48] for a complete account of these effects. The systematic error quoted below covers the range found with all of these variations.

The fit parameters now permit us to shift the simulation results to the infinite-volume, continuum limit and to remove the effects of partial quenching - hence restoring unitarity in the quark masses. Complete details of the procedure are outlined in Ref. [48]. The results are displayed in Fig. 9, where we observe a remarkable result. The tremendous spread of data seen in Fig. 8 is dramatically reduced, with all 80 points now lying very accurately on a universal curve.

The curve through Fig. 9 displays the determined variation of the $\rho$-meson mass with pion mass. This curve also presents an extrapolation to the physical point, allowing extraction of the physical $\rho$-meson mass

$$
M_{\rho}=778(4)_{-6}^{+16}(8) \mathrm{MeV} \text {, }
$$

where the first error is statistical, the second is systematic and the third from the determination of $\Lambda$ [48]. This result is in excellent agreement with the experimentally observed mass.

\subsection{The role of strangeness in the nucleon}

One of the major experimental successes in hadron physics in recent years has been the development of the capacity to map out the flavor composition of the electromagnetic form factors of the nucleon. Parity-violating electron scattering (PVES) is an essential tool in this program. Exposing the role of the strange quark via these measurements 
provides direct information on the underlying dynamics of nonperturbative QCD - a compelling achievement both experimentally and theoretically. The most precise separation of the strange electric and magnetic form factors is available at $\mathrm{Q}^{2} \simeq$ $0.1 \mathrm{GeV} 2$, where experiments by the SAMPLE [52, 53], PVA4 [54] and HAPPEx [55,56] collaborations have been performed with varying kinematics and targets. At higher $\mathrm{Q}^{2}$, HAPPEx [57, 58], PVA4 [59] and the forward angle G0 experiment [60] provide further information over the range $\mathrm{Q}^{2} \sim 0.12-1.0 \mathrm{GeV}^{2}$.

In a recent global analysis of all this data, Young et al. [61] were able to establish an accurate range for the strangeness electric and magnetic form factors of the nucleon at $\mathrm{Q}^{2}=0.1 \mathrm{GeV}^{2}$, without the need for theoretical input (other than the general assumption of charge symmetry) or for assumptions that any particular form factor could be ignored in a particular experiment.

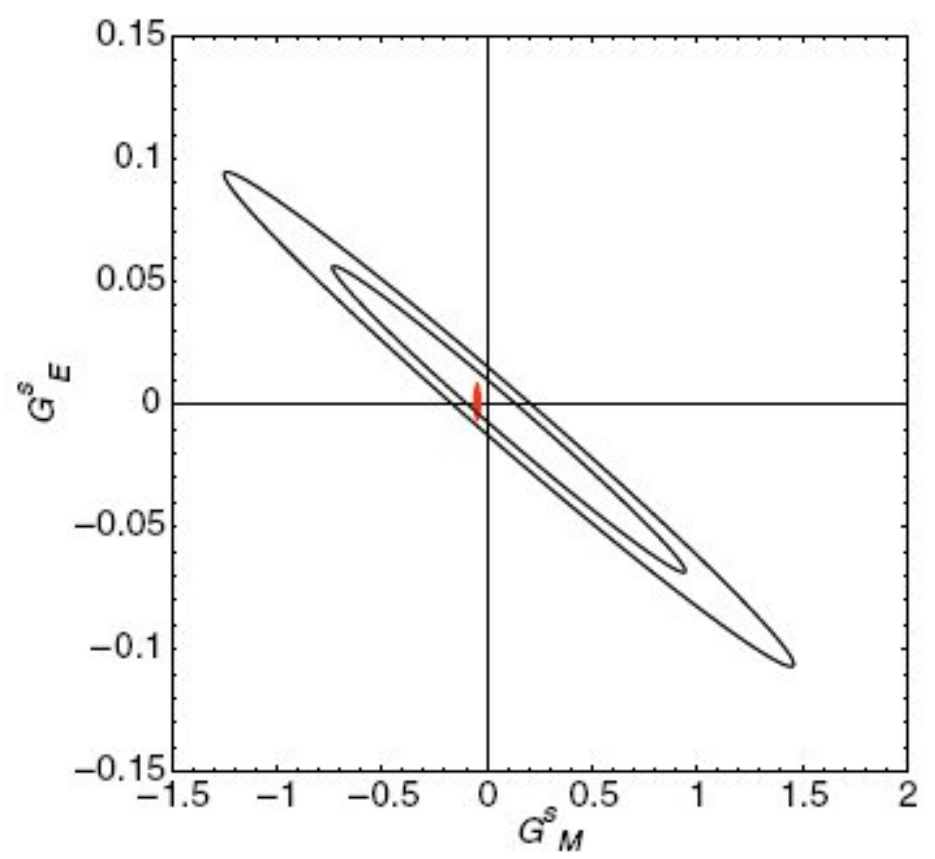

Fig. 10. The contours display the the 68 and $95 \%$ confidence intervals for the joint determination of $\mathrm{G}_{M}^{\mathrm{s}}$ and $\mathrm{G}_{E}^{\mathrm{s}}$ at $\mathrm{Q}_{2}=0.1 \mathrm{GeV}^{2}$ - from Ref. [61]. The shaded ellipse depicts the theoretical results of Refs. $[62,63]$.

The results for $\mathrm{G}_{E}^{\mathrm{s}}$ versus $\mathrm{G}_{M}^{\mathrm{s}}$ are shown in Fig. 10, along with the result (small shaded ellipse) of recent lattice QCD simulations, supplemented by FRR chiral extrapolation [62, 63]. The level of agreement is excellent. It is even better if the most recent results from HAPPEx, as reported at the April meeting of the APS in Dallas, are also included in the data set. As this data is not yet published, we do not show the result but as presented it reduces the errors for both $\mathrm{G}_{E}^{\mathrm{s}}$ and $\mathrm{G}_{M}^{\mathrm{s}}$ by about a factor two with little movement from that shown in Fig. 10. At this level of precision the agreement with the predictions of non-perturbative QCD is truly remarkable! This represents the first accurate determination of a "disconnected" sea contribution to a hadron property.

The qualitative insight that this success gives us into hadron structure is even more interesting. In order to obtain the theoretical result the lattice data was "unquenched" 
using the techniques pioneered in Ref. [42] - which rely on FRR. This is yet another confirmation that chiral loops are strongly suppressed once the pion mass exceeds a mass scale of order the inverse of the hadron size, typically $0.4 \mathrm{GeV}$ for a baryon.

\section{Conclusion}

The electroweak interaction will remain a key tool for advancing nuclear physics for the foreseeable future. It will complement facilities based on hadronic probes, such as FAIR in Europe and J-PARC in Japan. With the $12 \mathrm{GeV}$ Upgrade at Jefferson Lab, it will be the only electromagnetic facility worldwide capable of meeting the scientific demands required to understand how QCD works in the non-perturbative regime. We mention especially its ability to map the spectrum of exotic mesons in the mass range 1.5 to 2.5 $\mathrm{GeV}$ and hence to address the origin of confinement - one of the top ten questions for 21st century science. It will also permit us to map out the precise distribution of charge and current within the nucleons and the distribution of momentum, spin and orbital angular momentum on the valence quarks. With respect to real nuclei, Jefferson Lab will be critical for unravelling the flavor and spin dependence of the EMC effect.

It is also worth noting the exciting synergy that exists between the experimental capabilities Jefferson Lab at $12 \mathrm{GeV}$ will provide and our growing capacity to use lattice QCD to calculate the unambiguous consequences of non-perturbative QCD. We have outlined some of the issues and especially the recent successes in the battle to unambiguously extract these consequences. Only through this combined effort will we be able to answer the fundamental question of whether QCD is the complete theory of the strong interaction, as many believe it is, or whether it is just a good start!

The $12 \mathrm{GeV}$ Upgrade at Jefferson Lab is an essential step towards establishing a new paradigm for nuclear physics which unifies our understanding of nuclear systems over otherwise impossible ranges of density and strangeness in terms of the best candidate for a fundamental theory of the strong force, QCD. Finally, in our quest to push beyond the Standard Model, precise experiments at lower energy with electroweak probes can test for new physics at the multi-TeV scale.

I would like to thank A. Lung and L. Harwood for providing details of the $12 \mathrm{GeV}$ project in a form suitable for the outline presented here. Much of the work presented here on chiral extrapolation was performed in collaboration with D. Leinweber and R. Young (and in the case of the $\rho$ meson with C. Allton and W. Armour) and it is a pleasure to acknowledge the many things they have taught me. The recent global analysis of parity violating electron scattering reported here was carried out in collaboration with $\mathrm{R}$. Young, R. Carlini and J. Roche. This work was supported by DOE contract DE-AC0584ER40150, under which SURA operates Jefferson Lab.

\section{References}

1. http://www.jlab.org/div dept/physics division/GeV/doe review/

2. http://www.jlab.org/div dept/physics division/GeV/doe review/CDR for Science Review.pdf

3. http://www.jlab.org/div dept/physics division/GeV/doe review/science review. pdf

4. W. Melnitchouk, A.W. Thomas, Phys. Lett. B 377, 11 (1996) [arXiv:nucl-th/9602038]

5. M. Botje, Eur. Phys. J. C 14, 285 (2000) 
6. X. Zheng et al. [Jefferson Lab Hall A Collaboration], Phys. Rev. C 70, 065207 (2004) [arXiv:nucl-ex/0405006]

7. C.E. Hyde-Wright, K. de Jager, Ann. Rev. Nucl. Part. Sci. 54, 217 (2004) [arXiv:nuclex/0507001]

8. X. Ji, Ann. Rev. Nucl. Part. Sci. 54, 413 (2004)

9. A.V. Belitsky, A.V. Radyushkin, Phys. Rept. 418, 1 (2005) [arXiv:hep-ph/0504030]

10. P. Hoodbhoy, X.D. Ji, W. Lu, Phys. Rev. D 59, 014013 (1999) [arXiv:hep-

$\mathrm{ph} / 9804337]$

11. J.J. Aubert et al. [European Muon Collaboration], Phys. Lett. B 123, 275 (1983)

12. D.F. Geesaman, K. Sait, A.W. Thomas, Ann. Rev. Nucl. Part. Sci. 45, 337 (1995)

13. I.C. Clo"et, W. Bentz, A.W. Thomas, Phys. Rev. Lett. 95, 052302 (2005) [arXiv:nuclth/0504019]

14. I.C. Cloet, W. Bentz, A.W. Thomas [arXiv:nucl-th/0605061]

15. J.R. Smith, G.A. Miller, Phys. Rev. C 72, 022203 (2005) [arXiv:nucl-th/0505048]

16. W. Bentz, A.W. Thomas, Nucl. Phys. A 696, 138 (2001) [arXiv:nucl-th/0105022]

17. P.A.M. Guichon, A.W. Thomas, Phys. Rev. Lett. 93, 132502 (2004) [arXiv:nucl-

th/0402064]

18. T. Lippert, S. Gusken, K. Schilling, Nucl. Phys. Proc. Suppl. 83 (2000) 182

19. C.W. Bernard et al., Phys. Rev. D 64, 054506 (2001) [arXiv:hep-lat/0104002]

20. L.F. Li, H. Pagels, Phys. Rev. Lett. 26, 1204 (1971)

21. T. Hatsuda, Phys. Rev. Lett. 65, 543 (1990)

134 The European Physical Journal Special Topics

22. S. Durr [arXiv:hep-lat/0208051]

23. R.E. Stuckey, M.C. Birse, J. Phys. G 23, 29 (1997) [arXiv:hep-ph/9602312]

24. G.P. Lepage [arXiv:nucl-th/9706029]

25. H. Pagels, Phys. Rept. 16, 219 (1975)

26. J. Gasser, M.E. Sainio, A. Svarc, Nucl. Phys. B 307, 779 (1988)

27. R.F. Lebed, Nucl. Phys. B 430, 295 (1994) [arXiv:hep-ph/9311234]

28. M.K. Banerjee, J. Milana, Phys. Rev. D 54, 5804 (1996) [arXiv:hep-ph/9508340]

29. S.V. Wright, Ph.D. Thesis (University of Adelaide, 2002)

30. A.W. Thomas, Adv. Nucl. Phys. 13, 1 (1984)

31. A. Ali Khan et al. [CP-PACS Collaboration], Phys. Rev. D 65, 054505 (2002)

[Erratum-ibid. D67, 059901 (2003)] [arXiv:hep-lat/0105015]

32. S. Aoki et al. [JLQCD Collaboration], Phys. Rev. D 68, 054502 (2003) [arXiv:heplat/0212039]

33. Y. Iwasaki, Nucl. Phys. B 258 (1985) 141

34. UKQCD, C.R. Allton et al., Phys. Rev. D 60 (1999) 034507 [hep-lat/9808016]

35. R. Sommer, Nucl. Phys. B 411 (1994) 839 [hep-lat/9310022]

36. R.G. Edwards, U.M. Heller, T.R. Klassen, Nucl. Phys. B 517 (1998) 377 [heplat/9711003]

37. R.D. Young, D.B. Leinweber, A.W. Thomas, Prog. Part. Nucl. Phys. 50, 399 (2003) [arXiv:hep-lat/0212031]

38. D.B. Leinweber, A.W. Thomas, R.D. Young, Phys. Rev. Lett. 92, 242002 (2004)

[arXiv:hep-lat/0302020]

39. R.D. Young, D.B. Leinweber, A.W. Thomas, Nucl. Phys. Proc. Suppl. 128, 227 (2004)

[arXiv:hep-lat/0311038]

40. C.R. Allton, W. Armour, D.B. Leinweber, A.W. Thomas, R.D. Young, Phys. Lett. B

628,125 
(2005) [arXiv:hep-lat/0504022]

41. D.B. Leinweber, A.W. Thomas, K. Tsushima, S.V. Wright, Phys. Rev. D 64, 094502 (2001)

42. R.D. Young, D.B. Leinweber, A.W. Thomas, S.V. Wright, Phys. Rev. D 66, 094507 (2002)

43. R.D. Young, D.B. Leinweber, A.W. Thomas, Phys. Rev. D 71, 014001 (2005)

44. G. Colangelo, S. Durr, Eur. Phys. J. C 33, 543 (2004)

45. S.R. Beane, Phys. Rev. D 70, 034507 (2004)

46. S.R. Beane, M.J. Savage, Phys. Rev. D 70, 074029 (2004)

47. G. Rupak, N. Shoresh, Phys. Rev. D 66, 054503 (2002)

48. W. Armour, C.R. Allton, D.B. Leinweber, A.W. Thomas, R.D. Young, Unified chiral analysis of the vector meson spectrum from lattice QCD, J. Phys. G, to appear [arXiv:hep-lat/0510078]

49. C.K. Chow, S.J. Rey, Nucl. Phys. B 528, 303 (1998)

50. M. Lublinsky, Phys. Rev. D 55, 249 (1997)

51. S. Aoki, Phys. Rev. D 68, 054508 (2003)

52. T.M. Ito et al. [SAMPLE Collaboration], Phys. Rev. Lett. 92, 102003 (2004)

53. D.T. Spayde et al. [SAMPLE Collaboration], Phys. Lett. B 583, 79 (2004)

54. F.E. Maas et al., Phys. Rev. Lett. 94, 152001 (2005)

55. K.A. Aniol et al. [HAPPEX Collaboration], Phys. Rev. Lett. 96, 022003 (2006)

56. K.A. Aniol et al. [HAPPEX Collaboration], Phys. Lett. B 635, 275 (2006)

57. K.A. Aniol et al. [HAPPEX Collaboration], Phys. Lett. B 509, 211 (2001)

58. K.A. Aniol et al. [HAPPEX Collaboration], Phys. Rev. C 69, 065501 (2004)

59. F.E. Maas et al. [A4 Collaboration], Phys. Rev. Lett. 93, 022002 (2004)

60. D.S. Armstrong et al. [G0 Collaboration], Phys. Rev. Lett. 95, 092001 (2005)

61. R.D. Young, J. Roche, R.D. Carlini, A.W. Thomas [arXiv:nucl-ex/0604010]

62. D.B. Leinweber et al., Phys. Rev. Lett. 94, 212001 (2005)

63. D.B. Leinweber et al. [arXiv:hep-lat/0601025] 